\title{
Nonlinear Delay Discrete Inequalities and Their Applications to Volterra Type Difference Equations
}

\author{
Yu Wu, ${ }^{1}$ Xiaopei Li, ${ }^{2}$ and Shengfu Deng ${ }^{3}$ \\ ${ }^{1}$ Yibin University, Yibin, Sichuan 644007, China \\ ${ }^{2}$ Department of Mathematics, Zhanjiang Normal University, Zhanjiang, Guangdong 524048, China \\ ${ }^{3}$ Institute of Applied Physics and Computational Mathematics, P.O. Box 8009, Beijing 100088, China
}

Correspondence should be addressed to Shengfu Deng, sf_deng@sohu.com

Received 7 September 2009; Accepted 14 January 2010

Academic Editor: Abdelkader Boucherif

Copyright (C) 2010 Yu Wu et al. This is an open access article distributed under the Creative Commons Attribution License, which permits unrestricted use, distribution, and reproduction in any medium, provided the original work is properly cited.

Delay discrete inequalities with more than one nonlinear term are discussed, which generalize some known results and can be used in the analysis of various problems in the theory of certain classes of discrete equations. Application examples to show boundedness and uniqueness of solutions of a Volterra type difference equation are also given.

\section{Introduction}

Gronwall-Bellman inequalities and their various linear and nonlinear generalizations play very important roles in the discussion of existence, uniqueness, continuation, boundedness, and stability properties of solutions of differential equations and difference equations. The literature on such inequalities and their applications is vast. For example, see [1-12] for continuous cases, and [13-20] for discrete cases. In particular, the book [21] written by Pachpatte considered three types of discrete inequalities:

$$
\begin{gathered}
u(n) \leq a(n)+\sum_{s=0}^{n-1} f(s) w(u(s)), \\
u^{2}(n) \leq a(n)+2 \sum_{s=0}^{n-1} f(s) u(s), \\
u^{2}(n) \leq a(n)+\sum_{s=0}^{n-1} f(s) w(u(s)) .
\end{gathered}
$$


In this paper, we consider a delay discrete inequality

$$
u(n) \leq a(n)+\sum_{i=1}^{m} \sum_{s=b_{i}(0)}^{b_{i}(n-1)} f_{i}(n, s) w_{i}(u(s)), \quad n \in \mathbf{N}_{0}
$$

which has $m$ nonlinear terms where $\mathbf{N}_{0}=\{0,1,2, \ldots\}$. We will show that many discrete inequalities like (1.1) can be reduced to this form. Our main result can be applied to analyze properties of solutions of discrete equations. We also give examples to show boundedness and uniqueness of solutions of a Volterra type difference equation.

\section{Main Results}

Assume that

$\left(C_{1}\right) a(n)$ is nonnegative for $n \in \mathbf{N}_{0}$ and $a(0)>0$;

$\left(C_{2}\right) b_{i}(n)(i=1, \ldots, m)$ are nondecreasing for $n \in \mathbf{N}_{0}$, the range of each $b_{i}$ belongs to $\mathbf{N}_{0}$, and $b_{i}(n) \leq n$

$\left(C_{3}\right)$ all $f_{i}(n, j)(i=1, \ldots, m)$ are nonnegative for $n, j \in \mathbf{N}_{0}$;

$\left(C_{4}\right)$ all $w_{i}(i=1, \ldots, m)$ are continuous and nondecreasing functions on $[0, \infty)$ and are positive on $(0, \infty)$. They satisfy the relationship $w_{1} \propto w_{2} \alpha \cdots \alpha w_{m}$ where $w_{i} \propto w_{i+1}$ means that $\left(w_{i+1}\right) / w_{i}$ is nondecreasing on $(0, \infty)$ (see [10]).

Let $W_{i}(u)=\int_{u_{i}}^{u}\left(d z / w_{i}(z)\right)$ for $u \geq u_{i}$ where $u_{i}>0$ is a given constant. Then, $W_{i}$ is strictly increasing so its inverse $W_{i}^{-1}$ is well defined, continuous, and increasing in its corresponding domain. Define $b_{i}(-1)=-1, \Delta u(n)=u(n+1)-u(n)$ and $\Delta_{2} r(n, j)=r(n, j+1)-r(n, j)$.

Theorem 2.1. Suppose that $\left(C_{1}\right)-\left(C_{4}\right)$ hold and $u(n)$ is a nonnegative function for $n \in \mathbf{N}_{0}$ satisfying (1.2). Then

$$
u(n) \leq W_{m}^{-1}\left[W_{m}(\tilde{a}(0))+\sum_{s=b_{m}(0)}^{b_{m}(n-1)} \tilde{f}_{m}(n, s)+\sum_{s=0}^{n-1} \frac{\Delta_{2} r_{m}(n, s)}{\phi_{m}\left(W_{m-1}^{-1}\left(r_{m}(0, s)\right)\right)}\right], \quad n \leq N_{1},
$$

where $\tilde{a}(n)=\max _{0 \leq \tau \leq n, \tau \in \mathbf{N}_{0}} a(\tau), \tilde{f}_{i}(n, j)=\max _{0 \leq \tau \leq n, \tau \in N_{0}} f_{i}(\tau, j), r_{m}(n, j)$ is determined recursively by

$$
\begin{gathered}
r_{1}(n, j)=\tilde{a}(j), \\
r_{i+1}(n, j)=W_{i}\left(r_{1}(n, 0)\right)+\sum_{s=b_{i}(0)}^{b_{i}(j-1)} \tilde{f}_{i}(n, s)+\sum_{s=0}^{j-1} \frac{\Delta_{2} r_{i}(n, s)}{\phi_{i}\left(W_{i-1}^{-1}\left(r_{i}(0, s)\right)\right)}, \quad i=1, \ldots, m-1,
\end{gathered}
$$

$\phi_{i}(u)=w_{i}(u) / w_{i-1}(u), \phi_{1}(u)=w_{1}(u), W_{0}=I$ (Identity), and $N_{1}$ is the largest positive integer such that

$$
W_{i}(\tilde{a}(0))+\sum_{s=b_{i}(0)}^{b_{i}\left(N_{1}-1\right)} \tilde{f}_{i}\left(N_{1}, s\right)+\sum_{s=0}^{N_{1}-1} \frac{\Delta_{2} r_{i}\left(N_{1}, s\right)}{\phi_{i}\left(W_{i-1}^{-1}\left(r_{i}(0, s)\right)\right)} \leq \int_{u_{i}}^{\infty} \frac{d z}{w_{i}(z)}, \quad i=1, \ldots, m .
$$


Remark 2.2. (1) $N_{1}$ is defined by (2.3) and $N_{1}=\infty$ when all $w_{i}(i=1, \ldots, m)$ satisfy $\int_{u_{i}}^{\infty}\left(d z / w_{i}(z)\right)=\infty$. Different choices of $u_{i}$ in $W_{i}$ do not affect our results (see [2]).

(2) If $b_{i}(n)=n$ for $i=1, \ldots, m$, then (2.1) gives the estimate of the following inequality:

$$
u(n) \leq a(n)+\sum_{i=1}^{m} \sum_{s=0}^{n-1} f_{i}(n, s) w_{i}(u(s)), \quad n \in \mathbf{N}_{0}
$$

by replacing $b_{m}(n-1), b_{m}(0), b_{i}(j-1), b_{i}(0)$, and $b_{i}\left(N_{1}-1\right)$ with $n-1,0, j-1,0$ and $N_{1}-1$, respectively. Especially, if $b_{1}(n)=n$ and $f_{1}(n, s)=f(s)$, then (1.2) for $m=1$ becomes the first inequality of (1.1). Equation (2.1) shows the same estimate given by $\left(b_{1}\right)$ of Theorem 4.2.3 in the book [21].

Lemma 2.3. $\Delta_{2} r_{i}(n, j)$ is nonnegative and nondecreasing in $n$, and $r_{i}(n, j)$ is nonnegative and nondecreasing in $n$ and $j$ for $i=1, \ldots, m$.

Proof. By the definitions of $\tilde{a}(n)$ and $\tilde{f}_{i}(n, j)$, it is easy to check that they are nonnegative and nondecreasing in $n$, and $\tilde{a}(n) \geq a(n)$ and $\tilde{f}_{i}(n, j) \geq f_{i}(n, j)$ for each fixed $j$ where $i=1, \ldots, m$. $a(0)>0$ in $\left(C_{1}\right)$ implies that $\tilde{a}(n)>0$ for all $n \leq N_{1}$. Clearly,

$$
\begin{gathered}
\Delta_{2} r_{1}(n+1, j)-\Delta_{2} r_{1}(n, j)=0, \\
\Delta_{2} r_{2}(n+1, j)-\Delta_{2} r_{2}(n, j)=\tilde{f}_{1}\left(n+1, b_{1}(j)\right)-\tilde{f}_{1}\left(n, b_{1}(j)\right)+\frac{\Delta_{2} r_{1}(n+1, j)-\Delta_{2} r_{1}(n, j)}{w_{1}\left(r_{1}(0, j)\right)} \geq 0,
\end{gathered}
$$

where $r_{1}(0, j)=\tilde{a}(j)>0$ is used, which yields that $\Delta_{2} r_{1}(n, j)$ and $\Delta_{2} r_{2}(n, j)$ are nondecreasing in $n$. Assume that $\Delta_{2} r_{l}(n, j)$ is nondecreasing in $n$. Then

$$
\Delta_{2} r_{l+1}(n+1, j)-\Delta_{2} r_{l+1}(n, j)=\tilde{f}_{l}\left(n+1, b_{l}(j)\right)-\tilde{f}_{l}\left(n, b_{l}(j)\right)+\frac{\Delta_{2} r_{l}(n+1, j)-\Delta_{2} r_{l}(n, j)}{\phi_{l}\left(W_{l-1}^{-1}\left(r_{l}(0, j)\right)\right)} \geq 0
$$

which implies that $\Delta_{2} r_{l+1}(n, j)$ is nondecreasing in $n$. By induction, $\Delta_{2} r_{i}(n, j)(i=1, \ldots, m)$ are nondecreasing in $n$. Similarly, we can prove that they are nonnegative by induction again. Then $r_{i}(n, j)(i=1, \ldots, m)$ are nonnegative and nondecreasing in $n$ and $j$.

Proof of Theorem 2.1. Take any arbitrary positive integer $\tilde{n} \leq N_{1}$ and consider the auxiliary inequality

$$
u(n) \leq r_{1}(\tilde{n}, n)+\sum_{i=1}^{m} \sum_{s=b_{i}(0)}^{b_{i}(n-1)} \tilde{f}_{i}(\tilde{n}, s) w_{i}(u(s)), \quad n \leq \tilde{n} .
$$


Claim that $u(n)$ in (2.7) satisfies

$$
u(n) \leq W_{m}^{-1}\left[W_{m}\left(r_{1}(\tilde{n}, 0)\right)+\sum_{s=b_{m}(0)}^{b_{m}(n-1)} \tilde{f}_{\mathrm{m}}(\tilde{n}, s)+\sum_{s=0}^{n-1} \frac{\Delta_{2} r_{m}(\tilde{n}, s)}{\phi_{m}\left(W_{m-1}^{-1}\left(r_{m}(0, s)\right)\right)}\right]
$$

for $n \leq\left\{\tilde{n}, N_{2}\right\}$ where $N_{2}$ is the largest positive integer such that

$$
W_{i}\left(r_{1}(\tilde{n}, 0)\right)+\sum_{s=b_{i}(0)}^{b_{i}\left(N_{2}-1\right)} \tilde{f}_{i}(\tilde{n}, s)+\sum_{s=0}^{N_{2}-1} \frac{\Delta_{2} r_{i}(\tilde{n}, s)}{\phi_{i}\left(W_{i-1}^{-1}\left(r_{i}(0, s)\right)\right)} \leq \int_{u_{i}}^{\infty} \frac{d z}{w_{i}(z)}
$$

$i=1, \ldots, m$.

Before we prove (2.8), notice that $N_{1} \leq N_{2}$. In fact, $r_{i}(\tilde{n}, n), \Delta_{2} r_{i}(\tilde{n}, n)$, and $\tilde{f}_{i}(\tilde{n}, n)$ are nondecreasing in $\widetilde{n}$ by Lemma 2.3. Thus, $N_{2}$ satisfying (2.9) gets smaller as $\tilde{n}$ is chosen larger. In particular, $N_{2}$ satisfies the same $(2.3)$ as $N_{1}$ for $\tilde{n}=N_{1}$ if $r_{1}(\tilde{n}, 0)=\tilde{a}(0)$ is applied.

We divide the proof of (2.8) into two steps by using induction.

Step $1(m=1)$. Let $z(n)=\sum_{s=b_{1}(0)}^{b_{1}(n-1)} \tilde{f}_{1}(\widetilde{n}, s) w_{1}(u(s))$ for $n \leq \tilde{n}$ and $z(0)=0$. It is clear that $z(n)$ is nonnegative and nondecreasing. Observe that (2.7) is equivalent to $u(n) \leq r_{1}(\tilde{n}, n)+z(n)$ for $n \leq \tilde{n}$ and by assumptions $\left(C_{2}\right)$ and $\left(C_{4}\right)$ and Lemma 2.3,

$$
\begin{aligned}
\Delta z(n) & =\tilde{f}_{1}\left(\tilde{n}, b_{1}(n)\right) w_{1}\left(u\left(b_{1}(n)\right)\right) \leq \tilde{f}_{1}\left(\tilde{n}, b_{1}(n)\right) w_{1}\left(r_{1}\left(\tilde{n}, b_{1}(n)\right)+z\left(b_{1}(n)\right)\right) \\
& \leq \tilde{f}_{1}\left(\tilde{n}, b_{1}(n)\right) w_{1}\left(r_{1}(\tilde{n}, n)+z(n)\right) .
\end{aligned}
$$

Since $w_{1}$ is nondecreasing and $r_{1}(\tilde{n}, n)=\tilde{a}(n)>0$, we have

$$
\begin{aligned}
\frac{\Delta z(n)+\Delta_{2} r_{1}(\tilde{n}, n)}{w_{1}\left(r_{1}(\tilde{n}, n)+z(n)\right)} & \leq \tilde{f}_{1}\left(\tilde{n}, b_{1}(n)\right)+\frac{\Delta_{2} r_{1}(\tilde{n}, n)}{w_{1}\left(r_{1}(\tilde{n}, n)+z(n)\right)} \\
& \leq \tilde{f}_{1}\left(\tilde{n}, b_{1}(n)\right)+\frac{\Delta_{2} r_{1}(\tilde{n}, n)}{w_{1}\left(r_{1}(0, n)\right)} .
\end{aligned}
$$

Then

$$
\begin{aligned}
\int_{z(n)+r_{1}(\tilde{n}, n)}^{z(n+1)+r_{1}(\tilde{n}, n+1)} \frac{d \tau}{w_{1}(\tau)} & \leq \int_{z(n)+r_{1}(\tilde{n}, n)}^{z(n+1)+r_{1}(\tilde{n}, n+1)} \frac{d \tau}{w_{1}\left(z(n)+r_{1}(\tilde{n}, n)\right)} \\
& \leq \frac{\Delta z(n)+\Delta_{2} r_{1}(\tilde{n}, n)}{w_{1}\left(z(n)+r_{1}(\tilde{n}, n)\right)} \\
& \leq \tilde{f}_{1}\left(\tilde{n}, b_{1}(n)\right)+\frac{\Delta_{2} r_{1}(\tilde{n}, n)}{w_{1}\left(r_{1}(0, n)\right)}
\end{aligned}
$$


and so

$$
\begin{aligned}
\int_{z(0)+r_{1}(\tilde{n}, 0)}^{z(n)+r_{1}(\tilde{n}, n)} \frac{d \tau}{w_{1}(\tau)} & =\sum_{s=0}^{n-1} \int_{z(s)+r_{1}(\tilde{n}, s)}^{z(s+1)+r_{1}(\tilde{n}, s+1)} \frac{d \tau}{w_{1}(\tau)} \\
& \leq \sum_{s=0}^{n-1} \tilde{f}_{1}\left(\tilde{n}, b_{1}(s)\right)+\sum_{s=0}^{n-1} \frac{\Delta_{2} r_{1}(\tilde{n}, s)}{w_{1}\left(r_{1}(0, s)\right)} \\
& =\sum_{s=b_{1}(0)}^{b_{1}(n-1)} \tilde{f}_{1}(\tilde{n}, s)+\sum_{s=0}^{n-1} \frac{\Delta_{2} r_{1}(\tilde{n}, s)}{w_{1}\left(r_{1}(0, s)\right)}
\end{aligned}
$$

The definition of $W_{1}$ in Theorem 2.1 and $z(0)=0$ show

$$
W_{1}\left(z(n)+r_{1}(\tilde{n}, n)\right) \leq W_{1}\left(r_{1}(\tilde{n}, 0)\right)+\sum_{s=b_{1}(0)}^{b_{1}(n-1)} \tilde{f}_{1}(\tilde{n}, s)+\sum_{s=0}^{n-1} \frac{\Delta_{2} r_{1}(\tilde{n}, s)}{w_{1}\left(r_{1}(0, s)\right)}, \quad n \leq \tilde{n} .
$$

Equation (2.9) shows that the right side of (2.14) is in the domain of $W_{1}^{-1}$ for all $n \leq \tilde{n}$. Thus the monotonicity of $W_{1}^{-1}$ implies

$$
u(n) \leq z(n)+r_{1}(\tilde{n}, n) \leq W_{1}^{-1}\left[W_{1}\left(r_{1}(\tilde{n}, 0)\right)+\sum_{s=b_{1}(0)}^{b_{1}(n-1)} \tilde{f}_{1}(\tilde{n}, s)+\sum_{s=0}^{n-1} \frac{\Delta_{2} r_{1}(\tilde{n}, s)}{w_{1}\left(r_{1}(0, s)\right)}\right]
$$

for $n \leq \tilde{n}$; that is, $(2.8)$ is true for $m=1$.

Step $2(m=k+1)$. Assume that $(2.8)$ is true for $m=k$. Consider

$$
u(n) \leq r_{1}(\tilde{n}, n)+\sum_{i=1}^{k+1} \sum_{s=b_{i}(0)}^{b_{i}(n-1)} \tilde{f}_{i}(\tilde{n}, s) w_{i}(u(s)), \quad n \leq \tilde{n} .
$$

Let $z(n)=\sum_{i=1}^{k+1} \sum_{s=b_{i}(0)}^{b_{i}(n-1)} \tilde{f}_{i}(\tilde{n}, s) w_{i}(u(s))$ and $z(0)=0$. Then $z(n)$ is nonnegative and nondecreasing and satisfies $u(n) \leq r_{1}(\tilde{n}, n)+z(n)$ for $n \leq \tilde{n}$. Moreover, we have

$$
\Delta z(n)=\sum_{i=1}^{k+1} \tilde{f}_{i}\left(\tilde{n}_{,} b_{i}(n)\right) w_{i}\left(u\left(b_{i}(n)\right)\right) \leq \sum_{i=1}^{k+1} \tilde{f}_{i}\left(\tilde{n}, b_{i}(n)\right) w_{i}\left(r_{1}\left(\tilde{n}, b_{i}(n)\right)+z\left(b_{i}(n)\right)\right)
$$


Since $w_{i}$ and $r_{1}$ are nondecreasing in their arguments and $r_{1}(\tilde{n}, n)>0$, we have by the assumption $b_{i}(n) \leq n$

$$
\begin{aligned}
\frac{\Delta z(n)+\Delta_{2} r_{1}(\tilde{n}, n)}{w_{1}\left(z(n)+r_{1}(\tilde{n}, n)\right)} \leq & \frac{\sum_{i=1}^{k+1} \tilde{f}_{i}\left(\tilde{n}, b_{i}(n)\right) w_{i}\left(z\left(b_{i}(n)\right)+r_{1}\left(\tilde{n}, b_{i}(n)\right)\right)}{w_{1}\left(z(n)+r_{1}(\tilde{n}, n)\right)}+\frac{\Delta_{2} r_{1}(\tilde{n}, n)}{w_{1}\left(r_{1}(\tilde{n}, n)\right)} \\
\leq & \tilde{f}_{1}\left(\tilde{n}, b_{1}(n)\right)+\sum_{i=2}^{k+1} \tilde{f}_{i}\left(\tilde{n}, b_{i}(n)\right) \frac{w_{i}\left(z\left(b_{i}(n)\right)+r_{1}\left(\tilde{n}, b_{i}(n)\right)\right)}{w_{1}\left(z\left(b_{i}(n)\right)+r_{1}\left(\tilde{n}, b_{i}(n)\right)\right)}+\frac{\Delta_{2} r_{1}(\tilde{n}, n)}{w_{1}\left(r_{1}(0, n)\right)} \\
& \leq \tilde{f}_{1}\left(\tilde{n}, b_{1}(n)\right)+\sum_{i=1}^{k} \tilde{f}_{i+1}\left(\tilde{n}, b_{i+1}(n)\right) \tilde{\phi}_{i+1}\left(z\left(b_{i+1}(n)\right)+r_{1}\left(\tilde{n}, b_{i+1}(n)\right)\right) \\
& +\frac{\Delta_{2} r_{1}(\tilde{n}, n)}{w_{1}\left(r_{1}(0, n)\right)}
\end{aligned}
$$

for $n \leq \tilde{n}$ where $\widetilde{\phi}_{i+1}(u)=w_{i+1}(u) / w_{1}(u)$ for $i=1, \ldots, k$, which gives

$$
\begin{aligned}
\int_{z(n)+r_{1}(\tilde{n}, n)}^{z(n+1)+r_{1}(\tilde{n}, n+1)} \frac{d \tau}{w_{1}(\tau)} & \leq \int_{z(n)+r_{1}(\tilde{n}, n)}^{z(n+1)+r_{1}(\tilde{n}, n+1)} \frac{d \tau}{w_{1}\left(z(n)+r_{1}(\tilde{n}, n)\right)} \\
& \leq \frac{\Delta z(n)+\Delta_{2} r_{1}(\tilde{n}, n)}{w_{1}\left(r_{1}(\tilde{n}, n)+z(n)\right)} \\
& \leq \tilde{f}_{1}\left(\tilde{n}, b_{1}(n)\right)+\frac{\Delta_{2} r_{1}(\tilde{n}, n)}{w_{1}\left(r_{1}(0, n)\right)} \\
& +\sum_{i=1}^{k} \tilde{f}_{i+1}\left(\tilde{n}, b_{i+1}(n)\right) \tilde{\phi}_{i+1}\left(z\left(b_{i+1}(n)\right)+r_{1}\left(\tilde{n}, b_{i+1}(n)\right)\right)
\end{aligned}
$$

Therefore,

$$
\begin{aligned}
\int_{z(0)+r_{1}(\tilde{n}, 0)}^{z(n)+r_{1}(\tilde{n}, n)} \frac{d \tau}{w_{1}(\tau)} \leq & \sum_{s=b_{1}(0)}^{b_{1}(n-1)} \tilde{f}_{1}(\tilde{n}, s)+\sum_{s=0}^{n-1} \frac{\Delta_{2} r_{1}(\tilde{n}, s)}{w_{1}\left(r_{1}(0, s)\right)} \\
& +\sum_{i=1}^{k} \sum_{s=0}^{n-1} \tilde{f}_{i+1}\left(\widetilde{n}, b_{i+1}(s)\right) \tilde{\phi}_{i+1}\left(z\left(b_{i+1}(s)\right)+r_{1}\left(\tilde{n}, b_{i+1}(s)\right)\right),
\end{aligned}
$$

that is,

$$
\begin{aligned}
W_{1}\left(z(n)+r_{1}(\tilde{n}, n)\right) \leq & W_{1}\left(r_{1}(\tilde{n}, 0)\right)+\sum_{s=b_{1}(0)}^{b_{1}(n-1)} \tilde{f}_{1}(\tilde{n}, s)+\sum_{s=0}^{n-1} \frac{\Delta_{2} r_{1}(\tilde{n}, s)}{w_{1}\left(r_{1}(0, s)\right)} \\
& +\sum_{i=1}^{k} \sum_{s=b_{i+1}(0)}^{b_{i+1}(n-1)} \tilde{f}_{i+1}(\tilde{n}, s) \tilde{\phi}_{i+1}\left(z(s)+r_{1}(\tilde{n}, s)\right),
\end{aligned}
$$


or equivalently

$$
\xi(n) \leq c_{1}(\tilde{n}, n)+\sum_{i=1}^{k} \sum_{s=b_{i+1}(0)}^{b_{i+1}(n-1)} \tilde{f}_{i+1}(\tilde{n}, s) \tilde{\phi}_{i+1}\left(W_{1}^{-1}(\xi(s))\right), \quad n \leq \tilde{n}
$$

the same as (2.7) for $m=k$ where $\xi(n)=W_{1}\left(z(n)+r_{1}(\tilde{n}, n)\right)$ and

$$
c_{1}(\tilde{n}, n)=W_{1}\left(r_{1}(\tilde{n}, 0)\right)+\sum_{s=b_{1}(0)}^{b_{1}(n-1)} \tilde{f}_{1}(\tilde{n}, s)+\sum_{s=0}^{n-1} \frac{\Delta_{2} r_{1}(\tilde{n}, s)}{w_{1}\left(r_{1}(0, s)\right)}
$$

From the assumption $\left(C_{4}\right)$, each $\tilde{\phi}_{i+1}\left(W_{1}^{-1}\right), i=1, \ldots, k$, is continuous and nondecreasing on $[0, \infty)$ and is positive on $(0, \infty)$ since $W_{1}^{-1}$ is continuous and nondecreasing on $[0, \infty)$. Moreover, $\tilde{\phi}_{2}\left(W_{1}^{-1}\right) \propto \tilde{\phi}_{3}\left(W_{1}^{-1}\right) \propto \cdots \propto \tilde{\phi}_{k+1}\left(W_{1}^{-1}\right)$. By the inductive assumption, we have

$$
\xi(n) \leq \Phi_{k+1}^{-1}\left[\Phi_{k+1}\left(c_{1}(\tilde{n}, 0)\right)+\sum_{s=b_{k+1}(0)}^{b_{k+1}(n-1)} \tilde{f}_{k+1}(\tilde{n}, s)+\sum_{s=0}^{n-1} \frac{\Delta_{2} c_{k}(\tilde{n}, s)}{\psi_{k+1}\left(\Phi_{k}^{-1}\left(c_{k}(0, s)\right)\right)}\right]
$$

for $n \leq \min \left\{\tilde{n}, N_{3}\right\}$ where $\Phi_{i+1}(u)=\int_{\tilde{u}_{i+1}}^{u}\left(d z / \tilde{\phi}_{i+1}\left(W_{1}^{-1}(z)\right)\right), u>0, \Phi_{1}=I$ (Identity), $\tilde{u}_{i+1}=$ $W_{1}\left(u_{i+1}\right), \Phi_{i+1}^{-1}$ is the inverse of $\Phi_{i+1}, \psi_{i+1}(u)=\tilde{\phi}_{i+1}\left(W_{1}^{-1}(u)\right) / \tilde{\phi}_{i}\left(W_{1}^{-1}(u)\right)=w_{i+1}\left(W_{1}^{-1}(u)\right) /$ $w_{i}\left(W_{1}^{-1}(u)\right), i=1, \ldots, k$,

$$
c_{i+1}(\tilde{n}, n)=\Phi_{i+1}\left(c_{1}(\tilde{n}, 0)\right)+\sum_{s=b_{i+1}(0)}^{b_{i+1}(n-1)} \tilde{f}_{i+1}(\tilde{n}, s)+\sum_{s=0}^{n-1} \frac{\Delta_{2} c_{i}(\tilde{n}, s)}{\psi_{i+1}\left(\Phi_{i}^{-1}\left(c_{i}(0, s)\right)\right)},
$$

$i=1, \ldots, k-1$, and $N_{3}$ is the largest positive integer such that

$$
\begin{gathered}
\Phi_{i+1}\left(c_{1}(\tilde{n}, 0)\right)+\sum_{s=b_{i+1}(0)}^{b_{i+1}\left(N_{3}-1\right)} \tilde{f}_{i+1}(\tilde{n}, s)+\sum_{s=0}^{N_{3}-1} \frac{\Delta_{2} c_{i}(\tilde{n}, s)}{\psi_{i+1}\left(\Phi_{i}^{-1}\left(c_{i}(0, s)\right)\right)} \\
\leq \int_{\tilde{u}_{i+1}}^{W_{1}(\infty)} \frac{d z}{\tilde{\phi}_{i+1}\left(W_{1}^{-1}(z)\right)}, \quad i=1, \ldots, k .
\end{gathered}
$$


Note that

$$
\begin{aligned}
\Phi_{i}(u) & =\int_{\tilde{u}_{i}}^{u} \frac{d z}{\widetilde{\phi}_{i}\left(W_{1}^{-1}(z)\right)}=\int_{W_{1}\left(u_{i}\right)}^{u} \frac{w_{1}\left(W_{1}^{-1}(z)\right) d z}{w_{i}\left(W_{1}^{-1}(z)\right)} \\
& =\int_{u_{i}}^{W_{1}^{-1}(u)} \frac{d z}{w_{i}(z)}=W_{i} \circ W_{1}^{-1}(u), \quad i=2, \ldots, k+1, \\
\psi_{i+1}\left(\Phi_{i}^{-1}(u)\right) & =\frac{w_{i+1}\left(W_{1}^{-1}\left(\Phi_{i}^{-1}(u)\right)\right)}{w_{i}\left(W_{1}^{-1}\left(\Phi_{i}^{-1}(u)\right)\right)}=\frac{w_{i+1}\left(W_{1}^{-1}\left(W_{1}\left(W_{i}^{-1}(u)\right)\right)\right.}{w_{i}\left(W_{1}^{-1}\left(W_{1}\left(W_{i}^{-1}(u)\right)\right)\right)} \\
& =\frac{w_{i+1}\left(W_{i}^{-1}(u)\right)}{w_{i}\left(W_{i}^{-1}(u)\right)}=\phi_{i+1}\left(W_{i}^{-1}(u)\right), \quad i=1, \ldots, k+1 .
\end{aligned}
$$

Thus, we have from (2.24) that

$$
\begin{aligned}
u(n) & \leq r_{1}(\tilde{n}, n)+z(n)=W_{1}^{-1}(\xi(n)) \\
& \leq W_{k+1}^{-1}\left[W_{k+1}\left(W_{1}^{-1}\left(c_{1}(\tilde{n}, 0)\right)\right)+\sum_{s=b_{k+1}(0)}^{b_{k+1}(n-1)} \tilde{f}_{k+1}(\tilde{n}, s)+\sum_{s=0}^{n-1} \frac{\Delta_{2} c_{k}(\tilde{n}, s)}{\phi_{k+1}\left(W_{k}^{-1}\left(c_{k}(0, s)\right)\right)}\right] \\
& \leq W_{k+1}^{-1}\left[W_{k+1}\left(r_{1}(\tilde{n}, 0)\right)+\sum_{s=b_{k+1}(0)}^{b_{k+1}(n-1)} \tilde{f}_{k+1}(\tilde{n}, s)+\sum_{s=0}^{n-1} \frac{\Delta_{2} c_{k}(\tilde{n}, s)}{\phi_{k+1}\left(W_{k}^{-1}\left(c_{k}(0, s)\right)\right)}\right]
\end{aligned}
$$

for $n \leq \min \left\{\tilde{n}, N_{3}\right\}$ since $c_{1}(\tilde{n}, 0)=W_{1}\left(r_{1}(\tilde{n}, 0)\right)$.

In the following, we prove that $c_{i}(\widetilde{n}, n)=r_{i+1}(\widetilde{n}, n)$ by induction again. have

It is clear that $c_{1}(\tilde{n}, n)=r_{2}(\tilde{n}, n)$ for $i=1$. Suppose that $c_{l}(\tilde{n}, n)=r_{l+1}(\tilde{n}, n)$ for $i=l$. We

$$
\begin{aligned}
c_{l+1}(\tilde{n}, n) & =\Phi_{l+1}\left(c_{1}(\tilde{n}, 0)\right)+\sum_{s=b_{l+1}(0)}^{b_{l+1}(n-1)} \tilde{f}_{l+1}(\tilde{n}, s)+\sum_{s=0}^{n-1} \frac{\Delta_{2} c_{l}(\tilde{n}, s)}{\psi_{l+1}\left(\Phi_{l}^{-1}\left(c_{l}(0, s)\right)\right)} \\
& =W_{l+1}\left(r_{1}(\tilde{n}, 0)\right)+\sum_{s=b_{l+1}(0)}^{b_{l+1}(n-1)} \tilde{f}_{l+1}(\tilde{n}, s)+\sum_{s=0}^{n-1} \frac{\Delta_{2} r_{l+1}(\tilde{n}, s)}{\phi_{l+1}\left(W_{l}^{-1}\left(r_{l+1}(0, s)\right)\right)} \\
& =r_{l+2}(\tilde{n}, n),
\end{aligned}
$$

where $c_{1}(\tilde{n}, 0)=W_{1}\left(r_{1}(\tilde{n}, 0)\right)$ is applied. It implies that it is true for $i=l+1$. Thus, $c_{i}(\tilde{n}, n)=$ $r_{i+1}(\tilde{n}, n)$ for $i=1, \ldots, k$. 
Equation (2.26) becomes

$$
\begin{aligned}
& W_{i+1}\left(r_{1}(\tilde{n}, 0)\right)+\sum_{s=b_{i+1}(0)}^{b_{i+1}\left(N_{3}-1\right)} \tilde{f}_{i+1}(\tilde{n}, s)+\sum_{s=0}^{N_{3}-1} \frac{\Delta_{2} r_{i+1}(\tilde{n}, s)}{\phi_{i+1}\left(W_{i}^{-1}\left(r_{i+1}(0, s)\right)\right)} \\
& \leq \int_{\tilde{u}_{i+1}}^{W_{1}(\infty)} \frac{d z}{\widetilde{\phi}_{i+1}\left(W_{1}^{-1}(z)\right)}=\int_{\tilde{u}_{i+1}}^{W_{1}(\infty)} \frac{w_{1}\left(W_{1}^{-1}(z)\right)}{w_{i+1}\left(W_{1}^{-1}(z)\right)} d z=\int_{u_{i+1}}^{\infty} \frac{d z}{w_{i+1}(z)}
\end{aligned}
$$

for $i=1, \ldots, k$. It implies that $N_{2}=N_{3}$. Thus, (2.28) becomes

$$
u(n) \leq W_{k+1}^{-1}\left[W_{k+1}\left(r_{1}(\tilde{n}, 0)\right)+\sum_{s=b_{k+1}(0)}^{b_{k+1}(n-1)} \tilde{f}_{k+1}(\tilde{n}, s)+\sum_{s=0}^{n-1} \frac{\Delta_{2} r_{k+1}(\tilde{n}, s)}{\phi_{k+1}\left(W_{k}^{-1}\left(r_{k+1}(0, s)\right)\right)}\right]
$$

for $n \leq \tilde{n}$. It shows that (2.8) is true for $m=k+1$. Thus, the claim is proved.

Now we prove (2.1). Replacing $n$ by $\tilde{n}$ in (2.8), we have

$$
u(\tilde{n}) \leq W_{m}^{-1}\left[W_{m}\left(r_{1}(\tilde{n}, 0)\right)+\sum_{s=b_{m}(0)}^{b_{m}(\tilde{n}-1)} \tilde{f}_{m}(\tilde{n}, s)+\sum_{s=0}^{\tilde{n}-1} \frac{\Delta_{2} r_{m}(\tilde{n}, s)}{\phi_{m}\left(W_{m-1}^{-1}\left(r_{m}(0, s)\right)\right)}\right] .
$$

Since (2.8) is true for any $\tilde{n} \leq N_{1}$, we replace $\tilde{n}$ by $n$ and get

$$
u(n) \leq W_{m}^{-1}\left[W_{m}\left(r_{1}(n, 0)\right)+\sum_{s=b_{m}(0)}^{b_{m}(n-1)} \tilde{f}_{m}(n, s)+\sum_{s=0}^{n-1} \frac{\Delta_{2} r_{m}(n, s)}{\phi_{m}\left(W_{m-1}^{-1}\left(r_{m}(0, s)\right)\right)}\right] .
$$

This is exactly $(2.1)$ since $r_{1}(n, 0)=\tilde{a}(0)$. This proves Theorem 2.1 .

Remark 2.4. If $a(n)=0$ for all $n \in \mathbf{N}_{0}$, then $\tilde{a}(0)=0$. Let $r_{1, u_{1}}(n, j):=r_{1}(n, j)+u_{1}$ where $u_{1}>0$ is given in $W_{1}(u)=\int_{u_{1}}^{u}\left(d z / w_{1}(z)\right)$. Using the same arguments as in (2.11) where $r_{1}(n, j)$ is replaced with the positive $r_{1, u_{1}}(n, j)$, we have $\Delta_{2} r_{1, u_{1}}(\tilde{n}, s)=0$ and (2.14) becomes

$$
\begin{aligned}
W_{1}\left(z(n)+r_{1, u_{1}}(\tilde{n}, n)\right) & \leq W_{1}\left(r_{1, u_{1}}(\tilde{n}, 0)\right)+\sum_{s=b_{1}(0)}^{b_{1}(n-1)} \tilde{f}_{1}(\tilde{n}, s) \\
& =W_{1}\left(u_{1}\right)+\sum_{s=b_{1}(0)}^{b_{1}(n-1)} \tilde{f}_{1}\left(\tilde{n}_{,} s\right)=\sum_{s=b_{1}(0)}^{b_{1}(n-1)} \tilde{f}_{1}(\tilde{n}, s),
\end{aligned}
$$

that is,

$$
\begin{aligned}
u(n) & \leq z(n)+r_{1, u_{1}}(\tilde{n}, n)=z(n)+u_{1} \\
& \leq W_{1}^{-1}\left[\sum_{s=b_{1}(0)}^{b_{1}(n-1)} \tilde{f}_{1}(\tilde{n}, s)\right], \quad n \leq \tilde{n},
\end{aligned}
$$


which is the same as (2.15) with a complementary definition that $W_{1}(0)=0$. From (1) of Remark 2.2, the estimate of (2.35) is independent of $u_{1}$. Then we similarly obtain (2.1) and all $r_{i}$ are defined by the same formula (2.2) where we define $W_{i}(0)=0$ for $i=1, \ldots, m$.

\section{Some Corollaries}

In this section, we apply Theorem 2.1 and obtain some corollaries.

Assume that $\varphi \in C\left(\mathbf{R}_{+}, \mathbf{R}_{+}\right)$is a strictly increasing function with $\varphi(\infty)=\infty$ where $\mathbf{R}_{+}=[0, \infty)$. Consider the inequality

$$
\varphi(u(n)) \leq a(n)+\sum_{i=1}^{m} \sum_{s=b_{i}(0)}^{b_{i}(n-1)} f_{i}(n, s) w_{i}(u(s)), \quad n \in N_{0} .
$$

Corollary 3.1. Suppose that $\left(C_{1}\right)-\left(C_{4}\right)$ hold. If $u(n)$ in (3.1) is nonnegative for $n \in \mathbf{N}_{0}$, then

$$
u(n) \leq \varphi^{-1}\left[\widetilde{W}_{m}^{-1}\left(\widetilde{W}_{m}(\tilde{a}(0))+\sum_{s=b_{m}(0)}^{b_{m}(n-1)} \tilde{f}_{m}(n, s)+\sum_{s=0}^{n-1} \frac{\Delta_{2} r_{m}(n, s)}{\widehat{\phi}_{m}\left(\widetilde{W}_{m-1}^{-1}\left(r_{m}(0, s)\right)\right)}\right)\right]
$$

for $n \leq N_{1}$ where $\widetilde{W}_{i}(u)=\int_{u_{i}}^{u}\left(d z / w_{i}\left(\varphi^{-1}(z)\right)\right), \widetilde{W}_{i}^{-1}$ is the inverse of $\widetilde{W}_{i}, \widetilde{W}_{0}=I($ Identity $)$, $\widehat{\phi}_{i}(u)=\left(w_{i}\left(\varphi^{-1}(u)\right)\right) /\left(w_{i-1}\left(\varphi^{-1}(u)\right)\right), \widehat{\phi}_{1}(u)=w_{1}\left(\varphi^{-1}(u)\right)$, and other related functions are defined as in Theorem 2.1 by replacing $w_{i}(u)$ with $w_{i}\left(\varphi^{-1}(u)\right)$.

Proof. Let $\xi(n)=\varphi(u(n))$. Then (3.1) becomes

$$
\xi(n) \leq a(n)+\sum_{i=1}^{m} \sum_{s=b_{i}(0)}^{b_{i}(n-1)} f_{i}(n, s) w_{i}\left(\varphi^{-1}(\xi(s))\right), \quad n \in \mathbf{N}_{0} .
$$

Note that $w_{i}\left(\varphi^{-1}(u)\right)$ satisfy $\left(C_{4}\right)$ for $i=1, \ldots, m$. Using Theorem 2.1, we obtain the estimate about $\xi(n)$ by replacing $w_{i}(u)$ with $w_{i}\left(\varphi^{-1}(u)\right)$. Then use the fact that $u(n)=\varphi^{-1}(\xi(n))$ and we get Corollary 3.1.

$$
\begin{aligned}
& \text { If } \varphi(u)=u^{p} \text { where } p>0 \text {, then (3.1) reads } \\
& \qquad u^{p}(n) \leq a(n)+\sum_{i=1}^{m} \sum_{s=b_{i}(0)}^{b_{i}(n-1)} f_{i}(n, s) w_{i}(u(s)), \quad n \in \mathbf{N}_{0} .
\end{aligned}
$$

Directly using Corollary 3.1, we have the following result.

Corollary 3.2. Suppose that $\left(C_{1}\right)-\left(C_{4}\right)$ hold. If $u(n)$ in (3.4) is nonnegative for $n \in \mathbf{N}_{0}$, then

$$
u(n) \leq\left[\widetilde{W}_{m}^{-1}\left(\widetilde{W}_{m}(\tilde{a}(0))+\sum_{s=b_{m}(0)}^{b_{m}(n-1)} \tilde{f}_{m}(n, s)+\sum_{s=0}^{n-1} \frac{\Delta_{2} r_{m}(n, s)}{\widehat{\phi}_{m}\left(\widetilde{W}_{m-1}^{-1}\left(r_{m}(0, s)\right)\right)}\right)\right]^{1 / p}
$$


for $n \leq N_{1}$ where $\widetilde{W}_{i}(u)=\int_{u_{i}}^{u}\left(d z / w_{i}\left(z^{1 / p}\right)\right), \widetilde{W}_{i}$ is the inverse of $\widetilde{W}_{i}, \widetilde{W}_{0}=I($ Identity $), \widehat{\phi}_{i}(u)=$ $w_{i}\left(u^{1 / p}\right) / w_{i-1}\left(u^{1 / p}\right), \widehat{\phi}_{1}(u)=w_{1}\left(u^{1 / p}\right)$, and other related functions are defined as in Theorem 2.1 by replacing $w_{i}(u)$ with $w_{i}\left(u^{1 / p}\right)$.

If $m=1, p=2, b_{1}(n)=n,(3.4)$ becomes the second inequality of (1.1) with $f_{1}(n, s)=$ $2 f(s)$ and $w_{1}(u)=u$, and the third inequality of (1.1) with $f_{1}(n, s)=f(s)$ and $w_{1}(u)=w(u)$, which are discussed in the book [21]. Equation (3.5) yields the same estimates of Theorem 4.2.4 in the book [21].

\section{Applications to Volterra Type Difference Equations}

In this section, we apply Theorem 2.1 to study boundedness and uniqueness of solutions of a nonlinear delay difference equation of the form

$$
y(n)=\beta(n)+\sum_{s=b_{1}(0)}^{b_{1}(n-1)} F(n, s, y(s))+\sum_{s=b_{2}(0)}^{b_{2}(n-1)} H(n, s, y(s)), \quad n \in \mathbf{N}_{0},
$$

where $y: \mathbf{N}_{0} \rightarrow \mathbf{R}$ is an unknown function, $\beta$ maps from $\mathbf{N}_{0}$ to $\mathbf{R}, F$ and $H$ map from $\mathbf{N}_{0} \times \mathbf{N}_{0} \times \mathbf{R}$ to $\mathbf{R}$, and $b_{i}$ satisfies the assumption $\left(C_{2}\right)$ for $i=1,2$.

Theorem 4.1. Suppose that $\beta(0) \neq 0$ and the functions $F$ and $H$ in (4.1) satisfy the conditions

$$
\begin{gathered}
|F(n, s, y)| \leq f_{1}(n, s) \sqrt{|y|}, \\
|H(n, s, y)| \leq f_{2}(n, s)|y|,
\end{gathered}
$$

where $f_{1}, f_{2}: \mathbf{N}_{0} \times \mathbf{N}_{0} \rightarrow[0, \infty)$. If $y(n)$ is a solution of $(4.1)$ on $\mathbf{N}_{0}$, then

$$
|y(n)| \leq \tilde{a}(0) \exp \left[\sum_{s=b_{2}(0)}^{b_{2}(n-1)} \tilde{f}_{2}(n, s)+\sum_{s=0}^{n-1} \frac{\tilde{f}_{1}\left(n, b_{1}(s)\right)+(\Delta \tilde{a}(s) / \sqrt{\tilde{a}(s)})}{h(s)}\right],
$$

where

$$
\begin{gathered}
\tilde{a}(s)=\max _{0 \leq \tau \leq s, \tau \in \mathbf{N}_{0}}|\beta(\tau)|, \quad \tilde{f}_{1}(n, s)=\max _{0 \leq \tau \leq n, \tau \in N_{0}} f_{1}(\tau, s), \\
\tilde{f}_{2}(n, s)=\max _{0 \leq \tau \leq n, \tau \in \mathbf{N}_{0}} f_{2}(\tau, s), \\
h(n)=\sqrt{\tilde{a}(0)}+\frac{1}{2} \sum_{s=b_{1}(0)}^{b_{1}(n-1)} \tilde{f}_{1}(0, s)+\frac{1}{2} \sum_{s=0}^{n-1} \frac{\Delta \tilde{a}(s)}{\sqrt{\tilde{a}(s)}} .
\end{gathered}
$$


Proof. Using (4.1) and (4.2), the solution $y(n)$ satisfies

$$
u(n) \leq a(n)+\sum_{s=b_{1}(0)}^{b_{1}(n-1)} f_{1}(n, s) w_{1}(u(s))+\sum_{s=b_{2}(0)}^{b_{2}(n-1)} f_{2}(n, s) w_{2}(u(s)), \quad n \in \mathbf{N}_{0},
$$

where

$$
u(n)=|y(n)|, \quad a(n)=|\beta(n)|, \quad w_{1}(u)=\sqrt{u}, \quad w_{2}(u)=u
$$

Clearly, $\tilde{a}(n)>0$ for all $n \in N_{0}$ since $\beta(0) \neq 0$. For positive constants $u_{1}, u_{2}$, we have

$$
\begin{gathered}
W_{1}(u)=\int_{u_{1}}^{u} \frac{d z}{w_{1}(z)}=2\left(\sqrt{u}-\sqrt{u_{1}}\right), \quad W_{1}^{-1}(u)=\left(\frac{u}{2}+\sqrt{u_{1}}\right)^{2}, \\
W_{2}(u)=\int_{u_{2}}^{u} \frac{d z}{w_{2}(z)}=\ln \frac{u}{u_{2}}, \quad W_{2}^{-1}(u)=u_{2} \exp (u), \\
r_{1}(n, j)=\tilde{a}(j)>0, \quad r_{1}(n, 0)=\tilde{a}(0), \\
r_{2}(n, j)=2\left(\sqrt{\tilde{a}(0)}-\sqrt{u_{1}}\right)+\sum_{s=b_{1}(0)}^{b_{1}(j-1)} \tilde{f}_{1}(n, s)+\sum_{s=0}^{j-1} \frac{\Delta \tilde{a}(s)}{\sqrt{\tilde{a}(s)}}, \\
\Delta_{2} r_{2}(n, j)=\tilde{f}_{1}\left(n, b_{1}(j)\right)+\frac{\Delta \tilde{a}(j)}{\sqrt{\tilde{a}(j)}}, \quad \phi_{2}(u)=\frac{w_{2}(u)}{w_{1}(u)}=\sqrt{u} .
\end{gathered}
$$

It is obvious that $w_{1}$ and $w_{2}$ satisfy $\left(C_{4}\right)$. Applying Theorem 2.1 gives

$$
u(n) \leq \tilde{a}(0) \exp \left[\sum_{s=b_{2}(0)}^{b_{2}(n-1)} \tilde{f}_{2}(n, s)+\sum_{s=0}^{n-1} \frac{\tilde{f}_{1}\left(n, b_{1}(s)\right)+(\Delta \tilde{a}(s) / \sqrt{\tilde{a}(s)})}{h(s)}\right]
$$

which implies (4.3).

Theorem 4.2. Suppose that $\beta(0) \neq 0$ and the functions $F$ and $H$ in (4.1) satisfy the conditions

$$
\begin{gathered}
\left|F\left(n, s, y_{1}\right)-F\left(n, s, y_{2}\right)\right| \leq f_{1}(n, s) \sqrt{\left|y_{1}-y_{2}\right|}, \\
\left|H\left(n, s, y_{1}\right)-H\left(n, s, y_{2}\right)\right| \leq f_{2}(n, s)\left|y_{1}-y_{2}\right|
\end{gathered}
$$

where $f_{1}, f_{2}: \mathbf{N}_{0} \times \mathbf{N}_{0} \rightarrow[0, \infty)$. Then (4.1) has at most one solution on $\mathbf{N}_{0}$.

Proof. Let $y_{1}(n)$ and $y_{2}(n)$ be two solutions of (4.1) on $\mathbf{N}_{0}$. From (4.9), we have

$$
|u(n)| \leq \sum_{s=b_{1}(0)}^{b_{1}(n-1)} f_{1}(n, s) w_{1}(u(s))+\sum_{s=b_{2}(0)}^{b_{2}(n-1)} f_{2}(n, s) w_{2}(u(s)), \quad n \in \mathbf{N}_{0},
$$


where $u(n)=\left|y_{1}(n)-y_{2}(n)\right|, a(n)=0, w_{1}(u)=\sqrt{u}$ and $w_{2}(u)=u$. Appling Theorem 2.1, Remark 2.4, and the notation $W_{i}(0)=0$ for $i=1,2$, we obtain that $u(n)=0$ which implies that the solution is unique.

\section{Acknowledgments}

This paper was supported by Guangdong Provincial natural science Foundation (07301595). The authors would like to thank Professor Boling Guo for his great help.

\section{References}

[1] R. P. Agarwal, "On an integral inequality in $n$ independent variables," Journal of Mathematical Analysis and Applications, vol. 85, no. 1, pp. 192-196, 1982.

[2] R. P. Agarwal, S. Deng, and W. Zhang, "Generalization of a retarded Gronwall-like inequality and its applications," Applied Mathematics and Computation, vol. 165, no. 3, pp. 599-612, 2005.

[3] I. Bihari, "A generalization of a lemma of Bellman and its application to uniqueness problems of differential equations," Acta Mathematica Academiae Scientiarum Hungaricae, vol. 7, pp. 81-94, 1956.

[4] W.-S. Cheung, "Some new nonlinear inequalities and applications to boundary value problems," Nonlinear Analysis: Theory, Methods E Applications, vol. 64, no. 9, pp. 2112-2128, 2006.

[5] S. K. Choi, S. Deng, N. J. Koo, and W. Zhang, "Nonlinear integral inequalities of Bihari-type without class $H$," Mathematical Inequalities \& Applications, vol. 8, no. 4, pp. 643-654, 2005.

[6] L. Horváth, "Generalizations of special Bihari type integral inequalities," Mathematical Inequalities $\mathcal{E}$ Applications, vol. 8, no. 3, pp. 441-449, 2005.

[7] N. Lungu, “On some Gronwall-Bihari-Wendorff-type inequalities,” Fixed Point Theory, vol. 3, pp. 249_ 254, 2002.

[8] B. G. Pachpatte, "On generalizations of Bihari's inequality," Soochow Journal of Mathematics, vol. 31, no. 2, pp. 261-271, 2005.

[9] B. G. Pachpatte, "Integral inequalities of the Bihari type," Mathematical Inequalities E Applications, vol. 5, no. 4, pp. 649-657, 2002.

[10] M. Pinto, "Integral inequalities of Bihari-type and applications," Funkcialaj Ekvacioj, vol. 33, no. 3, pp. 387-403, 1990.

[11] H. Ye, J. Gao, and Y. Ding, "A generalized Gronwall inequality and its application to a fractional differential equation," Journal of Mathematical Analysis and Applications, vol. 328, no. 2, pp. 1075-1081, 2007.

[12] W. Zhang and S. Deng, "Projected Gronwall-Bellman's inequality for integrable functions," Mathematical and Computer Modelling, vol. 34, no. 3-4, pp. 393-402, 2001.

[13] W.-S. Cheung, "Some discrete nonlinear inequalities and applications to boundary value problems for difference equations," Journal of Difference Equations and Applications, vol. 10, no. 2, pp. 213-223, 2004.

[14] W.-S. Cheung and J. Ren, "Discrete non-linear inequalities and applications to boundary value problems," Journal of Mathematical Analysis and Applications, vol. 319, no. 2, pp. 708-724, 2006.

[15] S. Deng and C. Prather, "Nonlinear discrete inequalities of Bihari-type," submitted.

[16] B. G. Pachpatte, “On Bihari like integral and discrete inequalities," Soochow Journal of Mathematics, vol. 17, no. 2, pp. 213-232, 1991.

[17] V. N. Phat and J. Y. Park, "On the Gronwall inequality and asymptotic stability of nonlinear discrete systems with multiple delays," Dynamic Systems and Applications, vol. 10, no. 4, pp. 577-588, 2001.

[18] J. Popenda and R. P. Agarwal, "Discrete Gronwall inequalities in many variables," Computers $\mathcal{E}$ Mathematics with Applications, vol. 38, no. 1, pp. 63-70, 1999.

[19] L. Tao and H. Yong, "A generalization of discrete Gronwall inequality and its application to weakly singular Volterra integral equation of the second kind," Journal of Mathematical Analysis and Applications, vol. 282, no. 1, pp. 56-62, 2003. 
[20] F.-H. Wong, C.-C. Yeh, and C.-H. Hong, "Gronwall inequalities on time scales," Mathematical Inequalities $\mathcal{E}$ Applications, vol. 9, no. 1, pp. 75-86, 2006.

[21] B. G. Pachpatte, Integral and Finite Difference Inequalities and Applications, vol. 205 of North-Holland Mathematics Studies, Elsevier Science B.V., Amsterdam, The Netherlands, 2006. 